\title{
A New Strategy for Quality Evaluation and Identification of Representative Chemical Components in Polygonum multiflorum Thunb.
}

\author{
Yun-xia Li, ${ }^{1,2}$ Xiao-hong Gong, ${ }^{2}$ Mei-chen Liu, ${ }^{2}$ Cheng Peng, ${ }^{2}$ Peng Li, ${ }^{1}$ and Yi-tao Wang ${ }^{1}$ \\ ${ }^{1}$ Institute of Chinese Medical Sciences, State Key Laboratory of Quality Research in Chinese Medicine, University of Macau, Macau \\ ${ }^{2}$ Pharmacy College, Chengdu University of Traditional Chinese Medicine, State Key Laboratory Breeding Base of Systematic Research, \\ Development and Utilization of Chinese Medicine Resources, Chengdu 611137, China \\ Correspondence should be addressed to Cheng Peng; pengchengchengdu@126.com and Peng Li; pli1978@hotmail.com
}

Received 2 September 2016; Revised 12 December 2016; Accepted 29 December 2016; Published 24 January 2017

Academic Editor: Hong-Cai Shang

Copyright (C) 2017 Yun-xia Li et al. This is an open access article distributed under the Creative Commons Attribution License, which permits unrestricted use, distribution, and reproduction in any medium, provided the original work is properly cited.

\begin{abstract}
Polygonum multiflorum Thunb. (HSW) is widely used as herb medicine and health food additive. Recently, a series of HSW-induced hepatotoxicities have been reported and many studies have been carried out to investigate it. But contradictory conclusions were drawn that might be caused by the inconsistent quality of market decoction pieces. Therefore, the HSW decoction pieces quality was evaluated with a developed novel method in the paper. 25 batches of raw HSW (RHSW) and 21 batches of processed HSW (PHSW) samples were purchased from different provinces of China. HPLC determination was performed to identify and detect the contents of 16 chemical compounds in herbal material. Fingerprint similarity was analyzed using chromatography information and the results showed that most herbs were in good similarity. Then, a comprehensive evaluation strategy based on principal component analysis with representative quality control indicators was developed to evaluate the quality of HSW samples. And the rationality of the developed method was verified by HCA analysis. The results showed that the herb from Dabashan, Sichuan Province, no matter RHSW or PHSW had the best quality. Different representative components were selected for RHSW or PHSW decoction pieces which might be caused by the chemical reaction during processing. And most PHSW were unqualified according to the requirement of Chinese Pharmacopeia which might take the responsibility for the toxicity of HSW.
\end{abstract}

\section{Introduction}

The last decade has witnessed an emergence and rapid shift of the paradigm in chemotherapy, involving a gradual transition from monosubstance therapy that had long been advocated with great vehemence to multidrug therapy. As a result, herbal medicines (HMs) are widely used which contain multiple active constituents that synergistically act to elicit effects greater than individual components. The wide application brings great opportunities to HMs but it is also accompanied with big challenges in quality standard for the complex composition.

There are two trends in HMs quality evaluation. For a long time, the content detection of constituents is the main quality control method for HMs that marker compounds are chosen followed by qualitative and quantitative analysis. Therefore, the sample quality is easily to be classified as "qualified" or "unqualified" when the analytical data is directly compared with the presetting criteria. However, the selection of indicators and their content levels of the existing quality standard are often subjective or sometimes a little arbitrarily without powerful evidences $[1,2]$. With the development of modern analytical instrument, systematic evaluation emerges to include component information as much as possible $[3,4]$. Although the identification tells us what chemical compounds are contained in HMs, it is still hard to distinguish herb quality with such information.

Polygonum multiflorum Thunb. (He Shou Wu in Chinese pinyin, herein after referred to as HSW), the root of Polygonum multiflorum, is a famous traditional Chinese medicine and is also widely used in East Asia and North America with the name of Fo-ti. Modern pharmacological studies have 


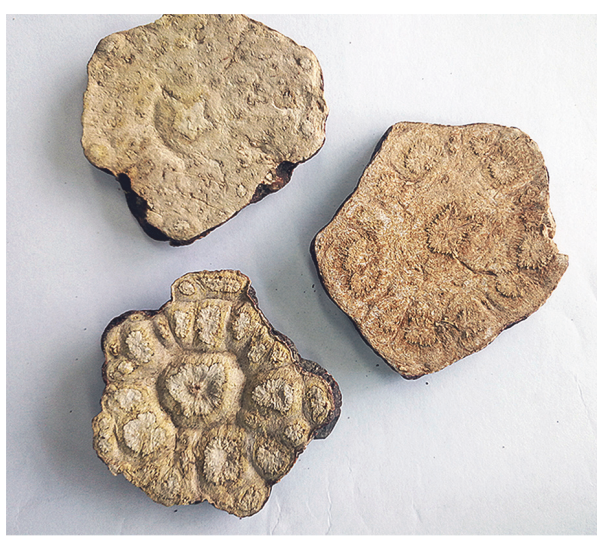

(a)

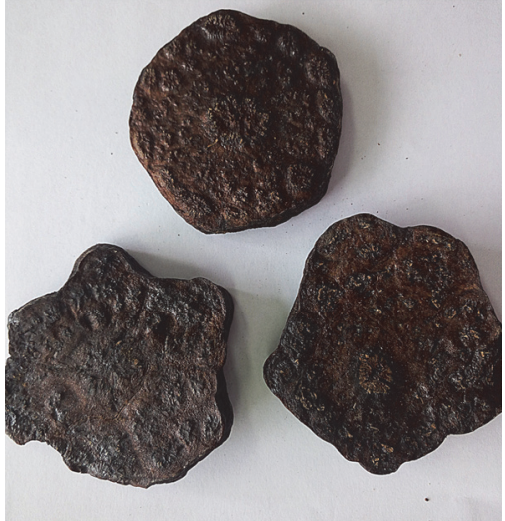

(b)

Figure 1: Picture of RHSW (a) decoction pieces and PHSW (b) decoction pieces.

shown that HSW has the effect of reducing blood lipid, protecting liver, enhancing immunity, improving memory, protecting nerve cells, antioxidation, antiaging, etc. [5]. With the significant biological and pharmacological effects, HSW has been used not only as a drug but also as a food supplement for many centuries in China. Two forms of HSW decoction pieces are available in market (Figure 1). One is raw HSW (RHSW) used for detoxification, eliminating carbuncle, preventing malaria, and relaxing bowel. Another is processed HSW (PHSW) used for nourishing liver and kidney, supplementing essence and blood, blackening hair, strengthening bones and muscles, eliminating dampness, and reducing lipid.

In 2002, RHSW and PHSW were authorized as health food additives by the government of People's Republic of China. Then, both of them were taken as tonic to prevent hair loss and premature graying in varies forms [6, 7]. However, a series of HSW-induced hepatotoxicities were reported in China, Korea, Japan, Britain, Italy, Australia, and other countries [8-10]. As a result, cautions on the intake of HSW preparations have been issued in China, Australia, Britain, Japan, and Canada.

However, people purchased the herb material directly from the market in most reported toxicity cases. The absence of quality control of market decoction pieces might be a key factor influencing HSW toxicity. As we know, various factors including geographical origin, harvesting, and processing influence herb quality which further results in different pharmacological effects $[11,12]$. Therefore, the comprehensive chemical composition analysis and sample classification are of great importance for the quality evaluation of HMs which has so far been multivariate and multi-index. To deal with the issue, an innovative comprehensive evaluation strategy based on principal component analysis with representative quality control indicators selection was developed to evaluate HSW quality. This approach of integrating the information of multiple indexes and using a comprehensive approach to evaluate the quality of HSW is an effective way to solve the problems of differences in the weights of index components and interactions among the components.

\section{Materials and Methods}

2.1. Chemicals and Reagents. Acetonitrile, methanol, formic acid, and water were of HPLC grade and purchased from Fisher Chemicals (Pittsburg, PA, USA). Gallic acid, procyanidins $B_{1}$, catechin, gallate, aloe emodin- 8 - $\beta$-o-glucoside, polydatin, stilbene glycosides, rhaponticin, resveratrol, emodin8- $\beta$-o-glucoside, physcion- 8 - $\beta$-o-glucoside, aloe emodin, rhein, chrysophanol, emodin, and physcion were purchased from National Institute for Food and Drug Control (Beijing, China) with purities above $98 \%$.

2.2. Plant Materials. 25 batches of RHSW and 21 batches of PHSW samples (the data were listed in the Supporting Information Table S1, in Supplementary Material available online at https://doi.org/10.1155/2017/6238464) were purchased from different provinces of China. All RHSW and PHSW samples were authenticated as the roots of Polygonum multiflorum Thunb. by Professor Jin Pei, and their voucher specimens were deposited at the Herbarium Center of Chengdu University of Traditional Chinese Medicine.

2.3. Preparation of Standard and Sample Solutions. 16 reference compounds were accurately weighed separately using Sartorius BP 211D electronic balance (Lower-Saxony, Germany) and dissolved in $10 \mathrm{~mL}$ volumetric flask with methanol to prepare stock solutions (gallic acid $131 \mu \mathrm{g} / \mathrm{mL}$, procyanidins B1 $98 \mu \mathrm{g} / \mathrm{mL}$, catechin $185 \mu \mathrm{g} / \mathrm{mL}$, gallate $142 \mu \mathrm{g} / \mathrm{mL}$, aloe emodin- 8 - $\beta$-o-glucoside $78 \mu \mathrm{g} / \mathrm{mL}$, polydatin $125 \mu \mathrm{g} / \mathrm{mL}$, stilbene glycosides $5400 \mu \mathrm{g} / \mathrm{mL}$, rhaponticin $142 \mu \mathrm{g} / \mathrm{mL}$, resveratrol $94 \mu \mathrm{g} / \mathrm{mL}$, emodin-8- $\beta$-o-glucoside $750 \mu \mathrm{g} / \mathrm{mL}$, physcion-8- $\beta$-o-glucoside $86 \mu \mathrm{g} / \mathrm{mL}$, aloe emodin $71 \mu \mathrm{g} / \mathrm{mL}$, rhein $81 \mu \mathrm{g} / \mathrm{mL}$, chrysophanol $500 \mu \mathrm{g} / \mathrm{mL}$, emodin $25 \mu \mathrm{g} / \mathrm{mL}$, and physcion $88 \mu \mathrm{g} / \mathrm{mL}$ ). Then, different volumes of stock solutions were mixed together to prepare serials of standard solutions with methanol (Table S2).

The samples powder (each about $0.25 \mathrm{~g}, n=3$ ) was soaked in $5 \mathrm{~mL} 50 \%$ aqueous ethanol for $30 \mathrm{~min}$ and then extracted by ultrasonication for $60 \mathrm{~min}$ at $40^{\circ} \mathrm{C}$. After being 
filtrated through a $0.45 \mu \mathrm{m}$ membrane filter, $5 \mu \mathrm{L}$ was injected for analysis.

2.4. HPLC Analysis. The analysis was performed using an Agilent 1260 series HPLC (Agilent) equipped with a quaternary pump, vacuum degasser, autosampler, and diode array detector. Chromatographic separation was carried out on an Agilent Zorbax XDB- $\mathrm{C}_{18}$ column $(5 \mu \mathrm{m}, 4.6 \mathrm{~mm} \times 250 \mathrm{~mm})$. The mobile phase consisted of acetonitrile (A) and water containing $0.1 \%$ formic acid (B) with a linear gradient elution at a flow rate of $1.0 \mathrm{~mL} / \mathrm{min}$. The gradient program was as follows: $5 \sim 10 \%$ A (0 5 min); 10 22\% A (5 30 $\mathrm{min}) ; 22 \sim 25 \%$ A (30 38 $\mathrm{min}) ; 25 \sim 32 \%$ A $(38 \sim 48 \mathrm{~min}) ; 32 \sim 45 \%$ A (48 $55 \mathrm{~min}) ; 45 \sim 85 \% \mathrm{~A}$ (55 65 $\mathrm{min})$; 85 95\% A (65 70 $\mathrm{min})$; and 95\% A (70 72 min). The detection wavelength was $275 \mathrm{~nm}$. The column temperature was maintained at $30^{\circ} \mathrm{C}$.

2.5. Method Validation. The detection wavelength was selected by DAD according to the number and height of peaks in the chromatograms of RHSW and PHSW extracts. The calibration curves were fitted to linear regression with a correlation coefficient $(>0.999)$ during the tested concentration ranges. The intra-assay and interassay precision were investigated by injecting standard solutions (three concentrations) six times in a day or in the consecutive three days. Reproducibility was studied through six independently prepared samples from a single batch of HSW. The stability test was performed by successively injecting the same sample solution over 24 hours.

2.6. Data Analysis. The chromatogram correlation coefficients among samples were calculated using the software "Similarity Evaluation System for Chromatographic Fingerprint of Traditional Chinese Medicine" (version 2004A) which was recommended by State Pharmacopeia Committee of the People's Republic of China. Principal component analysis (PCA) using Pareto Scaling method in preprocessing data and hierarchical cluster analysis (HCA) using Median clustering method in preprocessing data were performed by SPSS 21.0 (SPSS Inc., Chicago, USA).

Then, a novel evaluation strategy based on PCA with representative quality control indicators was developed to evaluate the quality of HSW samples. After PCA analysis was carried out on the detected chemical components, comprehensive index- integrated $F$ value $\left(F_{i}\right)$ was calculated as follows:

$$
A_{t f}=\frac{B_{t f}}{\sqrt{\lambda_{f}}}
$$

where $t$ referred to chemical component in the herb; $f$ referred to principal component; $A_{t f}$ was the contribution of component $t$ to principal component $f ; B_{t f}$ was standardized component $t$ contribution to principal component $f ; \lambda_{f}$ was eigenvalue of principal component $f$; and

$$
F_{i f}=\sum\left(A_{t f} \times C_{i t}\right)
$$

where $C_{i t}$ was the concentration of chemical compound $t$ in sample $i ; F_{i f}$ was the value of principal component $f$ in sample $i$.
The integrated $F_{i}$ value can be described as follows:

$$
F_{i}=\sum\left(F_{i f} \times V_{f}\right)
$$

where $V_{f}$ was variance of principal component to the total value.

The weight factor $\left(W_{t}\right)$ of component to the whole herb quality was calculated as follows:

$$
\begin{aligned}
W_{t} & =\sum\left(A_{t f} \times V_{f}\right), \\
W & =\sum W_{t}, \\
\text { \% of weight } & =\frac{W_{t}}{W} \times 100 \% .
\end{aligned}
$$

\section{Results and Discussion}

3.1. HPLC Method Validation. To gain high sensitivity and good peak capacity, the chromatographic conditions were optimized. Acetonitrile $/ 0.1 \%$ formic acid was used as the mobile phase to improve the retention behavior of the constituents on the HPLC column.

To detect more components at the same time, full scan was performed to optimize the wave length. The pretest results showed that both peak area and peak number at $275 \mathrm{~nm}$ provided more comprehensive profiles of RHSW and PHSW extracts. The relative standard deviations (RSDs) of the peaks were less than $2.0 \%$ for precision and $1.96 \%$ for reproducibility. The RSDs of the RPAs were less than $1.90 \%$ for stability. The recovery for the sixteen standards were 91.56 $109.58 \%$. All results indicated that the method was adequate and applicable (Tables S2 S5). Based on the above validated method, 16 constitutes chromatograms of RHSW and PHWS were visually distinguishable from each other and all peaks were simultaneously eluted within 72 minutes (Figure 2 ). The chemical constitute contents in RHSW and PHWS were listed in Tables 1 and 2.

3.2. Preparation of Sample Solutions. To extract as more components as possible, different factors were investigated in pretest including extraction solution $(0,20 \%, 40 \%, 50 \%$, $60 \%, 80 \%, 100 \%$ ethanol), extraction volume $(3,4,5,6,7 \mathrm{~mL})$, soak time $(10,20,30,40,50 \mathrm{~min})$, extraction temperature (20, 40,60 , and $\left.80^{\circ} \mathrm{C}\right)$, and extraction time $(10,20,40,60$, and $80 \mathrm{~min})$. Finally, the optimization extraction solution was as follows: $0.25 \mathrm{~g}$ samples powder was soaked in $5 \mathrm{~mL} 50 \%$ aqueous ethanol for $30 \mathrm{~min}$ and then extracted by ultrasonication for $60 \mathrm{~min}$ at $40^{\circ} \mathrm{C}$.

3.3. The Quality Evaluation of RHSW. Similarity Evaluation System for Chromatographic Fingerprint of TCM was widely applied in evaluating herb quality by calculating correlation coefficients based on peak area and retention time. The fingerprint similarities of RHSW were shown in Table 3 which ranged from 0.953 to 0.999 . Two exceptions were the herbs collected from Kunming city, Yunnan Province (number 1), which had a correlation coefficient of 0.48 , and those from Yancheng city, Jiangsu Province (numbers 13, 0.67). The two 


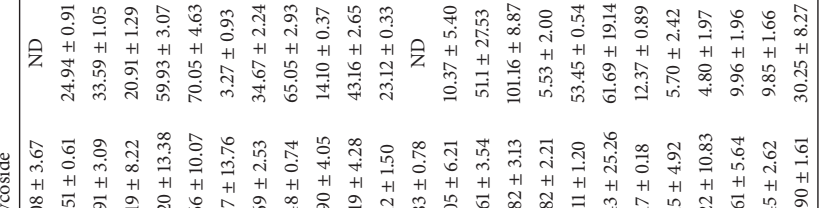

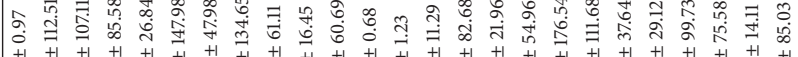

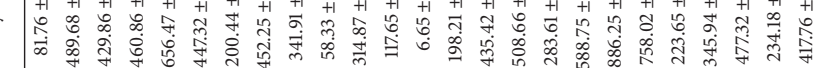

ए

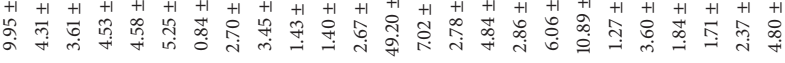

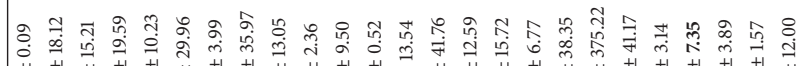

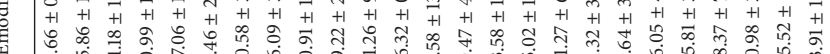

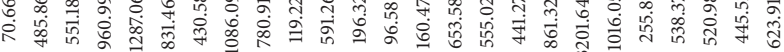

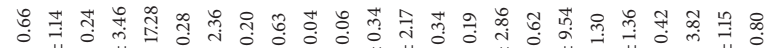

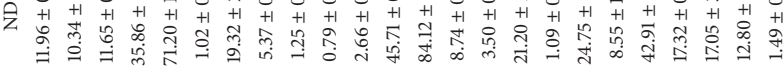

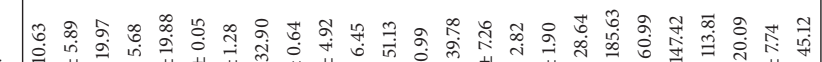

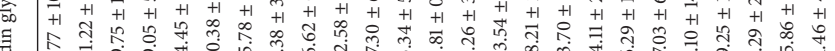

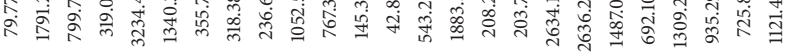

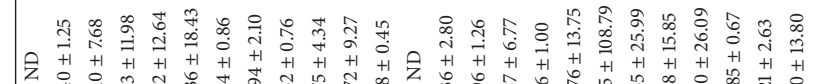

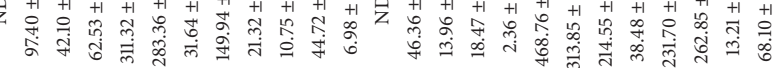

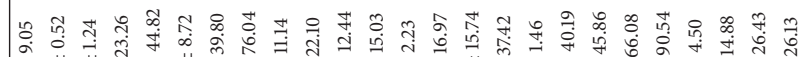

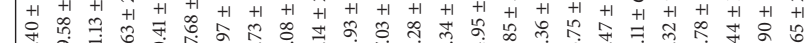

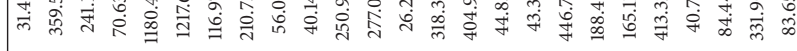

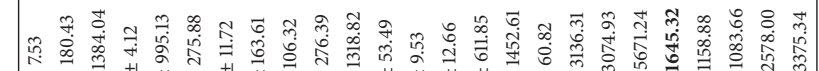

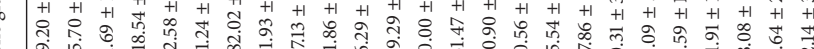

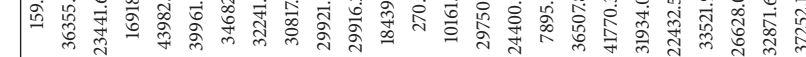
冈

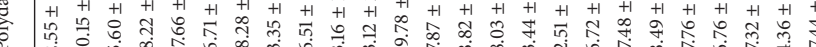

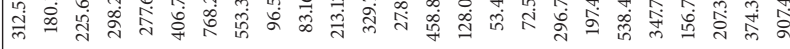

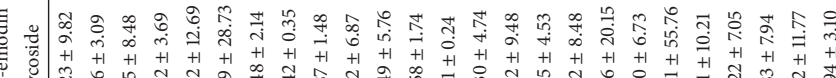

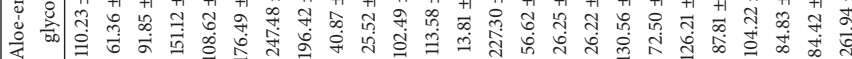

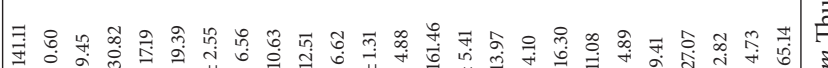

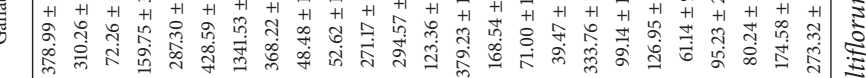

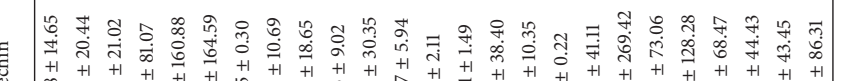

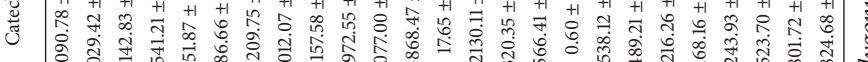

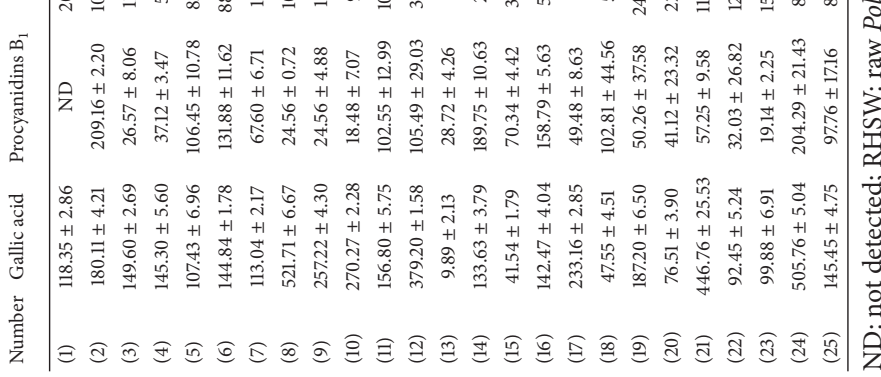




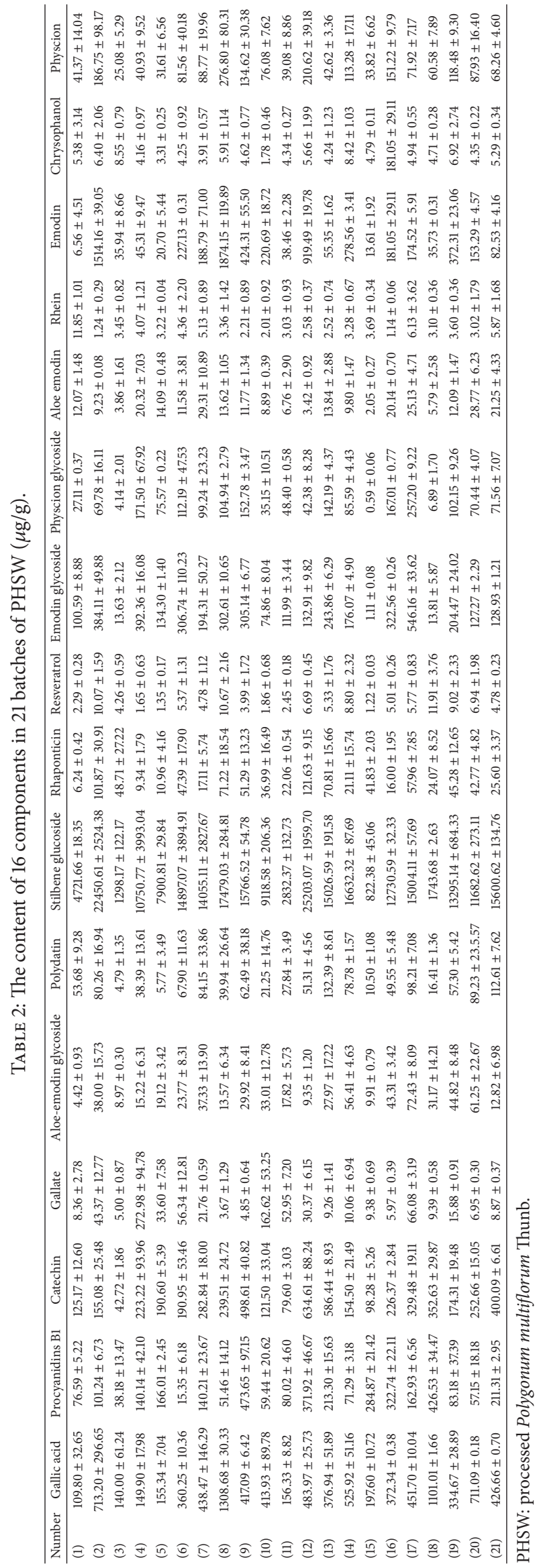




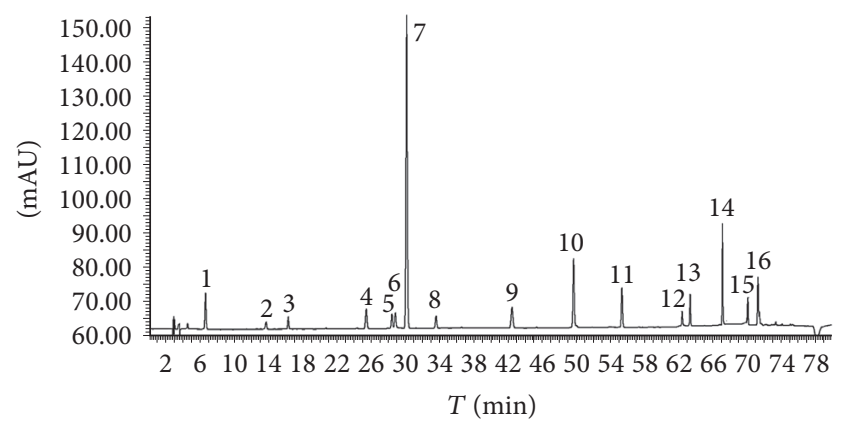

(a)

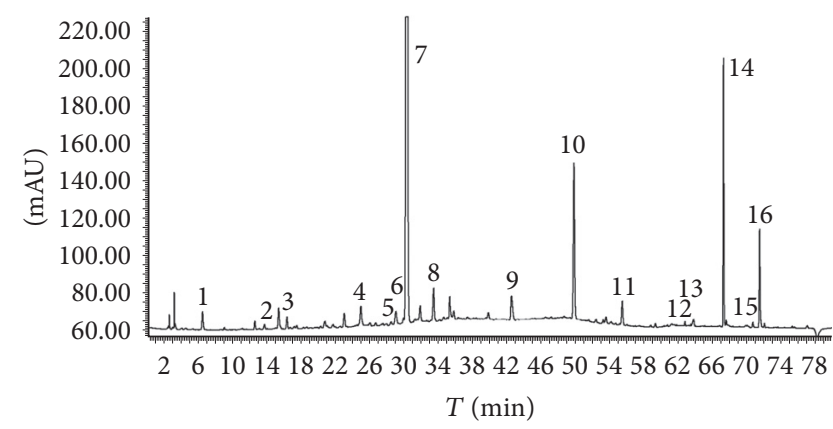

(b)

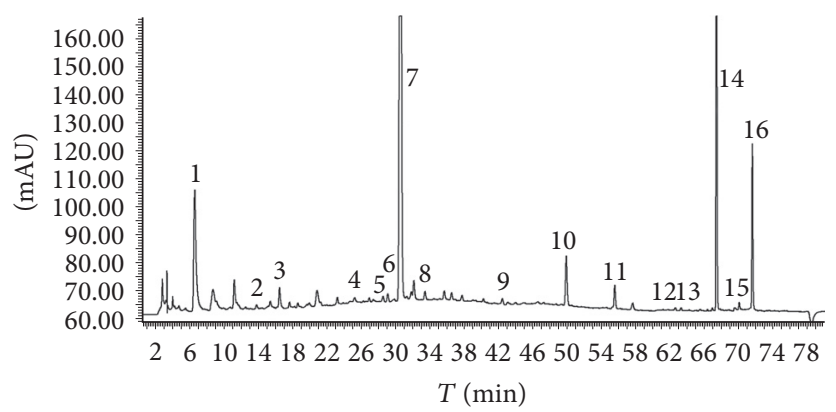

(c)

FiguRE 2: Chromatography of standard solution (a), RHSW (b) decoction pieces and PHSW (c) decoction pieces ((1) gallic acid, $t=6.62 \mathrm{~min}$; (2) procyanidins $\mathrm{B}_{1}, t=13.71 \mathrm{~min}$; (3) catechin, $t=16.28 \mathrm{~min}$; (4) gallate, $t=25.16 \mathrm{~min}$; (5) aloe emodin glycoside, $t=28.57$ min; (6) polydatin, $t=28.94 \mathrm{~min}$; (7) stilbene glucoside, $t=30.31 \mathrm{~min}$; (8) rhaponticin, $t=33.62 \mathrm{~min}$; (9) resveratrol, $t=42.49 \mathrm{~min}$; (10) emodin glycoside, $t=49.74 \mathrm{~min}$; (11) physcion glycoside, $t=55.31 \mathrm{~min}$; (12) aloe emodin, $t=62.35 \mathrm{~min}$; (13) rhein, $t=63.48 \mathrm{~min}$; (14) emodin, $t=67.13 \mathrm{~min}$; (15) chrysophanol, $t=70.17 \mathrm{~min}$; (16) physcion, $t=71.33 \mathrm{~min}$ ).

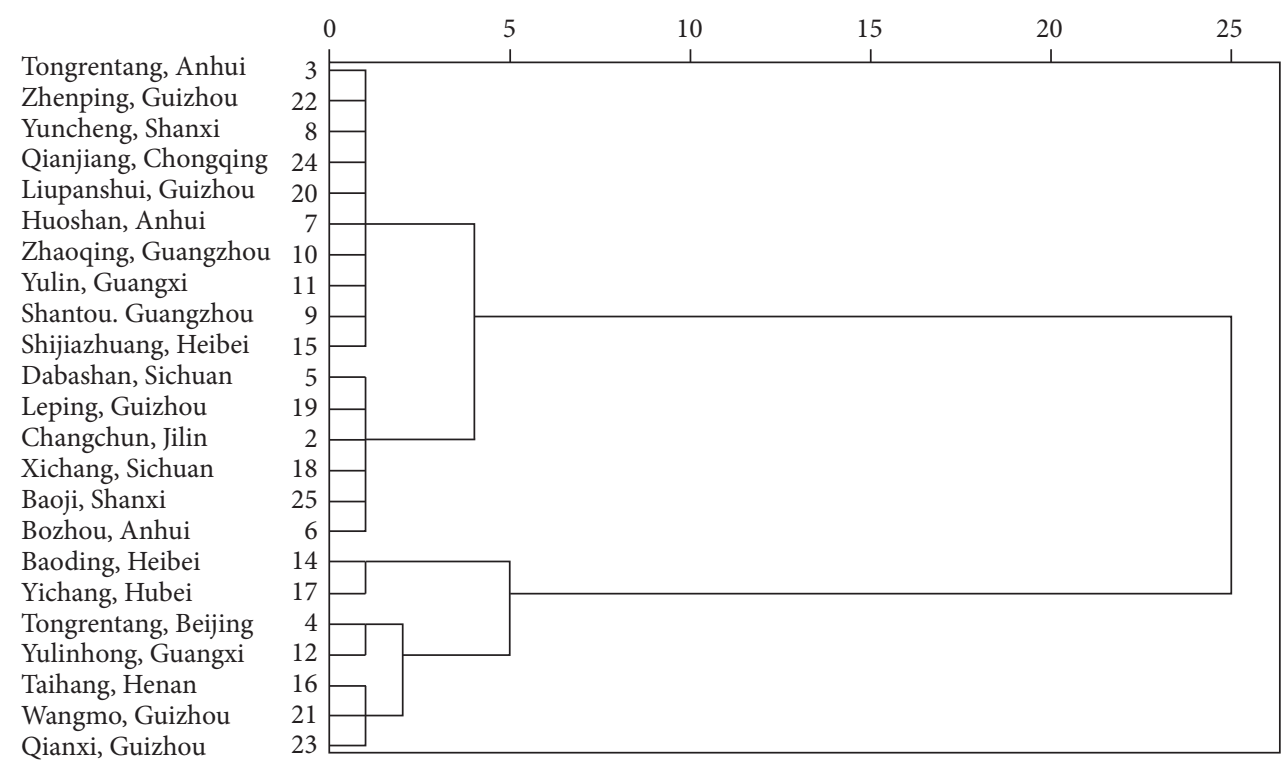

FIgURE 3: HCA analysis of RHSW decoction pieces with 16 components.

samples were excluded in HCA analysis which then classified 23 batches RHSW into five groups as in Figure 3.

However, the compounds concentration varied no matter in RHSW or PHSW (Tables 1 and 2) even if herb met similarity evaluation requirement of lager than 0.9 . It was impossible to evaluate the herb quality with so much information. HCA can classify the herb into different groups, but the result still cannot give us a conclusion which group had the best quality and so many chemical constitutes observed in the herb which can be chosen as the main components of the herb. 
TABLE 3: Fingerprint similarity for 25 batches of RHSW.

\begin{tabular}{lcc}
\hline Number & Collection location & Similarity degree \\
\hline$(1)$ & Kunming, Yunnan & 0.48 \\
$(2)$ & Changchun, Jilin & 0.995 \\
$(3)$ & Tongrentang, Anhui & 0.999 \\
$(4)$ & Tongrentang, Beijing & 0.994 \\
$(5)$ & Dabashan, Sichuan & 0.985 \\
$(6)$ & Bozhou, Anhui & 0.995 \\
$(7)$ & Huoshan, Anhui & 0.99 \\
$(8)$ & Yuncheng, Shanxi & 0.993 \\
$(9)$ & Shantou. Guangzhou & 0.997 \\
$(10)$ & Zhaoqing, Guangzhou & 0.994 \\
$(11)$ & Yulin, Guangxi & 0.999 \\
$(12)$ & Yulinhong, Guangxi & 0.977 \\
$(13)$ & Yancheng, Jiangsu & 0.67 \\
$(14)$ & Baoding, Heibei & 0.953 \\
$(15)$ & Shijiazhuang, Heibei & 0.99 \\
$(16)$ & Taihang, Henan & 0.997 \\
$(17)$ & Yichang, Hubei & 0.971 \\
$(18)$ & Xichang, Sichuan & 0.987 \\
$(19)$ & Leping, Guizhou & 0.982 \\
$(20)$ & Liupanshui, Guizhou & 0.99 \\
$(21)$ & Wangmo, Guizhou & 0.976 \\
$(22)$ & Zhenping, Guizhou & 0.994 \\
$(23)$ & Qianxi, Guizhou & 0.993 \\
$(24)$ & Qianjiang, Chongqing & 0.994 \\
$(25)$ & Baoji, Shanxi & 0.997 \\
\hline & &
\end{tabular}

RHSW: raw Polygonum multiflorum Thunb.

On the basis of chromatography, a two-step systematic strategy was developed to comprehensively evaluate the herb quality and select the representative compounds for the herb. PCA was a multivariate data analysis method to summarize massive numbers of variables in a dataset into a few correlated variables. In the study, PCA was firstly performed on the pretreated HPLC spectra of all RHSW samples. The PCA results showed that original sixteen variables (chemical compounds) were reduced to 6 principal components reflecting $82.33 \%$ of the influence of each compound on herb quality (Table 4). The contributions of each compound to principal components were listed in component matrix (Table 5).

The integrated $F$ value calculated according to "Materials and Methods" was shown in Table 6 which was in agreement with HCA classification. Group one (S14, S17) had the integrated $F$ value of 53.61 85.62; group two (S4, S12) had the $F$ value of 117.52 141.01; group three (S167, S21, S23) had the integrated $F$ value of 152.61 184.01; group four (S3, S7, S8, S9, S10, S11, S15, S20, S22, S24) had the integrated $F$ value of 193.14 228.93; group five (S2, S5, S6, S18, S19, S25) had the integrated $F$ value of 244.71 310.43. HCA result verified the reasonable of PCA analysis and indicated the integrated $F$ value can be used as index to evaluate the quality of RHSW.

In Chinese Pharmacopoeia (2015 edition), stilbene glycosides content in RHSW decoction was officially set as no less than $1.0 \%$ and the sum of the content of emodin- $8-\beta$ o-glucoside and physcion- 8 - $\beta$-o-glucoside was no less than $0.05 \%$. Table 1 showed that stilbene glycosides content in RHSW samples 1,13 , and 17 was $0.02 \%, 0.03 \%$, and $0.79 \%$, which was below the standard. The contents of emodin-8- $\beta$ o-glucoside and physcion-8- $\beta$-o-glucoside in RHSW samples $1,4,7,8,9,12,13,16$, and 17 was $0.01,0.03,0.04,0.04,0.03$, $0.02,0.01,0.02$, and $0.02 \%$. Sample S5 (integrated $F$ value $310.43)$ contained the highest stilbene glycosides $(2.20 \%)$ and emodin-8- $\beta$-o-glucoside and physcion- 8 - $\beta$-o-glucoside content $(1.64 \%)$ which also verified the conclusion that the integrated $F$ can represent the whole herb quality.

Although so many chemical compounds were detected in RHSW, which of them contributed most to the herb quality was still unknown. The peak area of components was used to present the contribution in some reports. However, the responsivity of each component was different at the same absorption length. Therefore, the same concentration might induce different peak area which caused error in calculation. In PCA, the data was firstly standardized to eliminate data error. The (\% of weight) value of each chemical compound was shown in Table 7. Component with high value (\% of weight) was chosen until the cumulative value achieved $80 \%$. In RHSW analysis, eight components were chosen including catechin, stilbene glycosides, rhaponticin, resveratrol, emodin-8- $\beta$-oglucoside, rhein, emodin, and physcion. Then, a second HCA of the herb was processed on the chosen 8 components (Figure 4). The analytical data was in accordance with the first HCA made with 16 components which verified the conclusion that the chosen chemicals can present the quality of the whole herb.

In previous herb quality control, the chemical with higher contents tended to be chosen as marker components. However, herb usually contained chemical components with similar structure. During the chemical synthesis, the contents of similar categories were correlated with each other. Therefore, it was unnecessary to analyze all chemicals in quality control. In the paper, the original 16 chemicals were simplified to 8 chemicals. The inclusion of physcion may be a reason for the exclusion of physcion-8- $\beta$-o-glucoside in the representative components.

3.4. The Quality Evaluation of PHSW. The same strategy was also applied to analyze the quality of 21 batches of PHWS samples from market. Fingerprints similarity of PHWS showed that 5 batches are below the required 0.9 (Table S6). Five principle components were selected which presented $81.25 \%$ information of PHSW (Table S7). The contributions of each compound in PHSW to principal components were listed in component matrix (Table S8). HCA analysis classified 21 batches of PHSW into four groups as in Figure S1. As shown in Table S9, PHSW group one (S1, S3, S11, S15, S18) had the integrated $F$ value of 6.50 36.52. Group two (S4, S5, S10, S16, S19, S20) had the integrated $F$ value of 59.79 102.84; group three (S6, S7, S8, S9, S13, S14, S17, S21) had the integrated $F$ value of 108.10 141.32; group four (S2, S12) had the integrated $F$ value of 175.15 191.12. Sample 12 form Dabashan, Sichuan Province, had the highest $F$ value of 191.12 which suggested it had the best quality. The interesting thing was that the best herb was 
TABLE 4: Principal component analysis of 25 batches of RHSW.

\begin{tabular}{lccc}
\hline Principal component & Eigenvalue & Variance contribution rate/\% & Cumulative contribution rate/\% \\
\hline$F_{1}$ & 5.096 & 31.852 & 31.852 \\
$F_{2}$ & 2.741 & 17.134 & 48.986 \\
$F_{3}$ & 1.722 & 10.763 & 59.749 \\
$F_{4}$ & 1.431 & 8.942 & 68.691 \\
$F_{5}$ & 1.238 & 7.738 & 76.429 \\
$F_{6}$ & 0.944 & 5.901 & 82.33 \\
\hline
\end{tabular}

RHSW: raw Polygonum multiflorum Thunb.

TABLE 5: Component loading matrix of 25 batches of RHSW.

\begin{tabular}{lcccccc}
\hline Component & $F_{1}$ & $F_{2}$ & $F_{3}$ & $F_{4}$ & $F_{5}$ & -0.035 \\
\hline Gallic acid & -0.488 & -0.037 & 0.188 & 0.431 & 0.679 \\
Procyanidins B1 & 0.06 & 0.206 & -0.413 & 0.736 & 0.177 & -0.159 \\
Catechin & -0.068 & 0.254 & 0.671 & 0.249 & 0.383 & -0.092 \\
Gallate & -0.054 & 0.814 & -0.193 & -0.049 & -0.064 \\
Aloe-emodin glycosides & -0.01 & 0.92 & 0.028 & 0.003 & -0.208 & -0.149 \\
Polydatin & -0.075 & 0.911 & 0.096 & -0.021 & -0.222 & 0.048 \\
Stilbene glucoside & 0.69 & 0.225 & -0.104 & -0.13 & -0.185 & 0.481 \\
Rhaponticin & 0.58 & 0.246 & -0.432 & 0.253 & 0.362 & 0.258 \\
Resveratrol & 0.814 & 0.141 & 0.138 & -0.2 & 0.083 & 0.062 \\
Emodin glycosides & 0.885 & 0.011 & -0.052 & -0.106 & 0.159 & -0.105 \\
Physcion glycosides & 0.768 & -0.092 & -0.483 & -0.18 & -0.011 & -0.024 \\
Aloe emodin & 0.594 & -0.286 & -0.178 & 0.351 & -0.158 & -0.079 \\
Rhein & 0.314 & 0.241 & 0.257 & -0.121 & 0.758 & -0.094 \\
Emodin & 0.711 & -0.074 & 0.539 & 0.104 & -0.303 & -0.235 \\
Chrysophanol & 0.588 & -0.054 & 0.25 & 0.566 & -0.116 \\
Physcion & 0.83 & -0.079 & 0.34 & -0.073 & -0.099 \\
\hline
\end{tabular}

RHSW: raw Polygonum multiflorum Thunb.

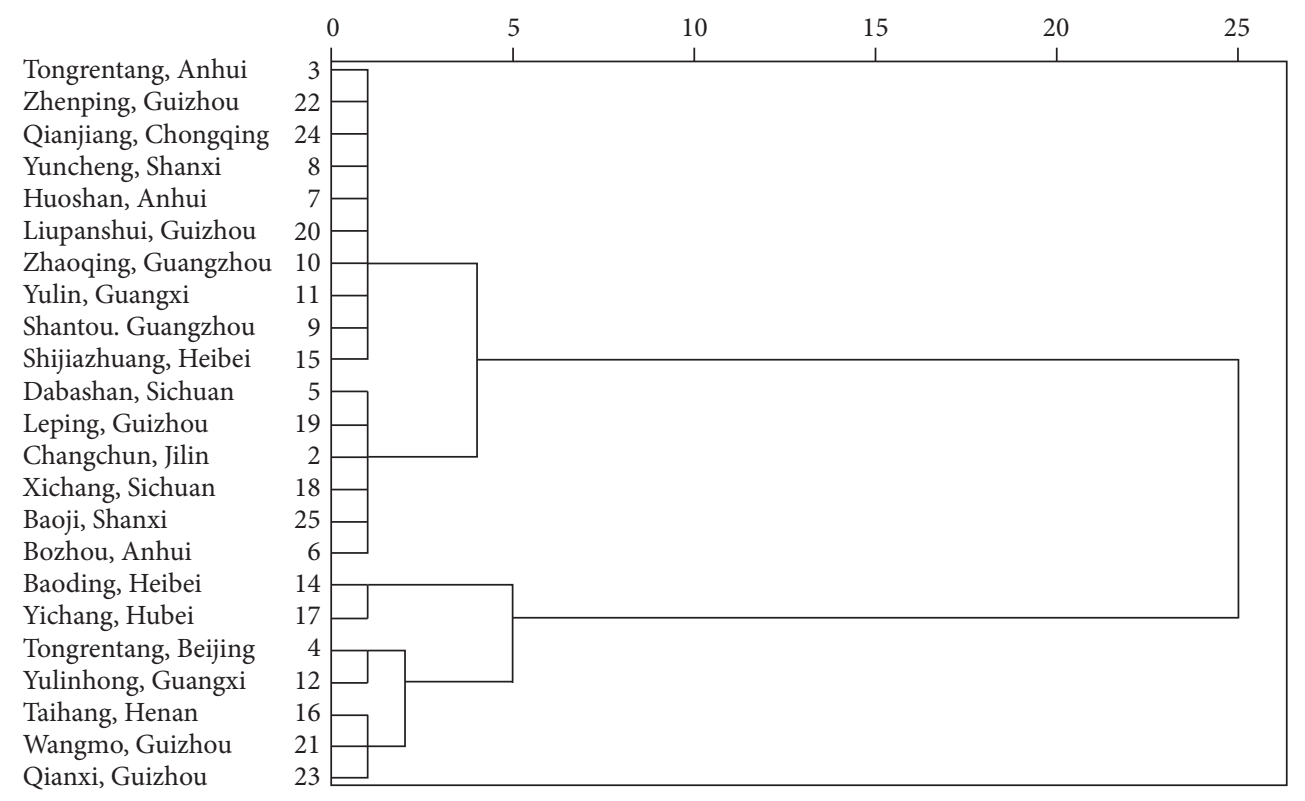

FIGURE 4: HCA analysis of RHSW decoction pieces with selected 8 representative components. 
TABLE 6: Integrated $F$ value of 25 batches of RHSW.

\begin{tabular}{|c|c|c|c|c|c|c|c|}
\hline Number & $F_{1}$ & $F_{2}$ & $F_{3}$ & $F_{4}$ & $F_{5}$ & $F_{6}$ & Integrated $F$ value \\
\hline (1) & 0.24 & 35.53 & 54.85 & 22.19 & 30.94 & -0.04 & 16.44 \\
\hline (2) & 616.25 & 267.29 & -117.87 & -189.07 & -276.41 & 908.37 & 244.71 \\
\hline (3) & 551.02 & 244.74 & -95.77 & -174.65 & -261.36 & 833.98 & 220.51 \\
\hline (4) & 291.29 & 132.01 & -31.00 & -91.90 & -147.79 & 425.47 & 117.52 \\
\hline (5) & 796.44 & 325.50 & -151.70 & -242.11 & -331.64 & 1095.05 & 310.43 \\
\hline (6) & 687.24 & 309.49 & -145.33 & -207.93 & -304.70 & 983.88 & 272.18 \\
\hline (7) & 546.77 & 304.84 & -105.02 & -181.52 & -284.62 & 859.42 & 227.54 \\
\hline (8) & 524.40 & 254.21 & -75.06 & -164.60 & -270.25 & 816.93 & 215.09 \\
\hline (9) & 494.95 & 219.97 & -73.63 & -155.45 & -247.63 & 773.87 & 200.02 \\
\hline (10) & 481.93 & 213.70 & -94.76 & -154.16 & -227.15 & 755.45 & 193.14 \\
\hline (11) & 493.75 & 224.95 & -85.81 & -152.93 & -233.84 & 746.15 & 198.84 \\
\hline (12) & 289.63 & 174.38 & 25.99 & -51.06 & -89.70 & 467.44 & 141.01 \\
\hline (13) & -0.27 & 2.44 & 2.43 & 2.76 & 2.25 & -7.73 & 0.56 \\
\hline (14) & 176.07 & 114.28 & 11.25 & -28.73 & -45.98 & 252.08 & 85.62 \\
\hline (15) & 524.26 & 211.64 & -111.53 & -168.12 & -234.83 & 741.38 & 201.79 \\
\hline (16) & 398.24 & 169.72 & -69.66 & -123.94 & -201.44 & 607.49 & 157.61 \\
\hline (17) & 137.05 & 55.14 & -20.87 & -41.88 & -71.38 & 203.74 & 53.61 \\
\hline (18) & 656.90 & 270.83 & -127.48 & -210.59 & -286.18 & 913.07 & 254.82 \\
\hline (19) & 768.42 & 303.84 & -35.08 & -227.74 & -331.74 & 1062.93 & 309.73 \\
\hline (20) & 552.91 & 252.52 & -43.32 & -164.41 & -237.30 & 801.13 & 228.93 \\
\hline (21) & 366.33 & 176.11 & -56.11 & -103.16 & -162.39 & 569.10 & 152.61 \\
\hline (22) & 559.68 & 245.12 & -89.96 & -179.89 & -257.44 & 840.30 & 224.16 \\
\hline (23) & 452.15 & 200.85 & -55.84 & -139.17 & -198.19 & 667.13 & 184.01 \\
\hline (24) & 527.36 & 245.34 & -104.34 & -160.03 & -259.82 & 829.94 & 213.34 \\
\hline (25) & 613.40 & 294.90 & -115.27 & -203.22 & -308.50 & 931.42 & 246.42 \\
\hline
\end{tabular}

RHSW: raw Polygonum multiflorum Thunb.

TABLE 7: Weight factor of component to the whole RHSW.

\begin{tabular}{|c|c|c|c|c|c|c|c|c|c|}
\hline Component & $F 1$ & $F 2$ & $F 3$ & $F 4$ & $F 5$ & F6 & $W_{t}$ & $W$ & $\%$ of weight \\
\hline Gallic acid & -0.216 & -0.022 & 0.143 & 0.360 & -0.032 & 0.699 & 1.376 & 147.360 & 0.933 \\
\hline Procyanidins $\mathrm{B}_{1}$ & 0.027 & 0.124 & -0.315 & 0.615 & 0.159 & -0.164 & 5.359 & & 3.637 \\
\hline Catechin & -0.030 & 0.153 & 0.511 & 0.208 & 0.344 & 0.013 & 11.777 & & 7.992 \\
\hline Gallate & -0.024 & 0.492 & -0.147 & -0.041 & -0.083 & -0.066 & 4.685 & & 3.179 \\
\hline Aloe-emodin glycoside & -0.004 & 0.556 & 0.021 & 0.003 & -0.187 & -0.153 & 7.281 & & 4.941 \\
\hline Polydatin & -0.033 & 0.550 & 0.073 & -0.018 & -0.200 & 0.049 & 7.750 & & 5.259 \\
\hline Stilbene glucoside & 0.306 & 0.136 & -0.079 & -0.109 & -0.166 & 0.495 & 11.875 & & 8.058 \\
\hline Rhaponticin & 0.257 & 0.149 & -0.329 & 0.212 & 0.325 & 0.266 & 13.161 & & 8.931 \\
\hline Resveratrol & 0.361 & 0.085 & 0.105 & -0.167 & 0.075 & 0.064 & 13.537 & & 9.186 \\
\hline Emodin glycosides & 0.392 & 0.007 & -0.040 & -0.089 & 0.143 & 0.108 & 13.124 & & 8.906 \\
\hline Physcion glycosides & 0.340 & -0.056 & -0.368 & -0.151 & -0.010 & -0.025 & 4.353 & & 2.954 \\
\hline Aloe emodin & 0.263 & -0.173 & -0.136 & 0.293 & -0.142 & -0.081 & 5.007 & & 3.398 \\
\hline Rhein & 0.139 & 0.146 & 0.196 & -0.101 & 0.681 & -0.097 & 12.830 & & 8.707 \\
\hline Emodin & 0.315 & -0.045 & 0.411 & 0.087 & -0.272 & 0.090 & 12.886 & & 8.745 \\
\hline Chrysophanol & 0.261 & -0.033 & 0.191 & 0.473 & -0.211 & -0.297 & 10.630 & & 7.214 \\
\hline Physcion & 0.368 & -0.048 & 0.259 & -0.061 & -0.104 & -0.102 & 11.730 & & 7.960 \\
\hline
\end{tabular}

RHSW: raw Polygonum multiflorum Thunb. 
from the same productive area (Dabashan, Sichuan province) no matter RHSW or PHSW which indicated the geography may be more suitable for HSW production.

Six components were chosen as the quality indicators for PHSW: aloe emodin-8- $\beta$-o-glucoside, polydatin, stilbene glycosides, emodin-8- $\beta$-o-glucoside, physcion-8- $\beta$-o-glucoside, and aloe emodin. The chosen components were not the same as those of RHSW. Then, a second HCA of the herb was processed on the chosen components (Figure S2). The analysis result was in accordance with the first HCA which verified the conclusion that the chosen components can present the quality of PHSW.

As for PHSW decoction, stilbene glycoside content was officially set as not less than $0.7 \%$ and the sum of the content of emodin and physcion was no less than $0.1 \%$. However, the concentration determination results indicated that stilbene glycoside in samples $1,3,11,15$, and 18 failed to meet the Chinese Pharmacopeia requirement with $s$ contents of $0.472 \%$, $0.13 \%, 0.28 \%, 0.08 \%$, and $0.64 \%$. Stilbene glycosides in samples 2 and 12 were higher than $2.0 \%$. And only the content of emodin and physcion in samples 2,8 , and 12 satisfied the standard of no less than $0.1 \%$ with $0.17 \%, 0.22 \%$, and $0.11 \%$. All the other samples had the content of $0.01 \% \sim 0.06 \%$ which was much lower than $0.1 \%$.

The situation may be caused by two factors. One reason might be the poor RHSW herb quality. But the result of quality evaluation suggested most RHSW quality was good enough to satisfy the requirement of Chinese Pharmacopeia. Another factor was processing technology in preparing PHSW. Both stilbene glycosides and anthraquinone compounds were unstable during processing. Long processing time resulted in the chemical change. In other reports, PHSW was always prepared for a long time, which not only resulted in the decreased content of stilbene glycosides and anthraquinone compounds but also induced the generation of hydroxymaltol, DDMP, and 5-HMF through Maillard reaction [13].

In summary, the study provided insights into the chemical profiles of RHSW and PHSW from different geographical origins. Systematic evaluation of RHSW and PHSW was successfully carried out after multistep data filtering and multivariate statistical analysis. Also, the proposed strategy integrating analysis might be a promising approach for the selection of representative component in the quality evaluation of HMs. Although the developed strategy was carried out on the identified chemical components in the study, it can also be applied in unidentified herb analysis to screen the useful information.

\section{Additional Points}

The supporting information is the resource of RHSW and PHSW; the method validation of RHSW and PHSW; the analytical procedure and HCA analysis of PHSW.

\section{Competing Interests}

The authors declare no competing financial interests.

\section{Authors' Contributions}

Yun-xia Li and Xiao-hong Gong conducted the experiments. Yun-xia Li and Xiao-hong Gong wrote the manuscript and prepared the figures. Xiao-hong Gong and Mei-chen Liu conducted the sample collection and data analysis. Cheng Peng, Peng Li, and Yi-tao Wang conceived the study. Yun-xia $\mathrm{Li}$ and Xiao-hong Gong contributed equally to this paper.

\section{Acknowledgments}

The study was supported by Project cofunded by China Ministry of Science and Technology and the Macao Science and Technology Development Fund (007/2014/AMJ); the Open Fund of the State Key Laboratory of Quality Research in Chinese Medicine (Grant no. 009); National Natural Science Foundation of China (81373943, 81573583); Sichuan Youth Science and Technology Foundation (2013JQ0018); Sichuan Provincial Science and Technology Department of Youth Science and Technology Innovation Research Team Program (2015TD0028, 2016TD0006, and 2017TD0001).

\section{References}

[1] X.-D. Ma, Y.-X. Fan, C.-C. Jin et al., "Specific targeted quantification combined with non-targeted metabolite profiling for quality evaluation of Gastrodia elata tubers from different geographical origins and cultivars," Journal of Chromatography A, vol. 1450, pp. 53-63, 2016.

[2] D. Yan, J. X. Li, Y. Xiong et al., "Promotion of quality standard of herbal medicine by constituent removing and adding," Scientific Reports, vol. 4, article 3668, 2014.

[3] E. J. Kim, J. Kwon, S. H. Park et al., "Metabolite profiling of angelica gigas from different geographical origins using $1 \mathrm{H}$ NMR and UPLC-MS analyses," Journal of Agricultural and Food Chemistry, vol. 59, no. 16, pp. 8806-8815, 2011.

[4] I. Bondia-Pons, O. Savolainen, R. Törrönen, J. A. Martinez, K. Poutanen, and K. Hanhineva, "Metabolic profiling of Goji berry extracts for discrimination of geographical origin by nontargeted liquid chromatography coupled to quadrupole timeof-flight mass spectrometry," Food Research International, vol. 63, pp. 132-138, 2014.

[5] L. F. Lin, B. Ni, H. M. Lin et al., "Traditional usages, botany, phytochemistry, pharmacology and toxicology of Polygonum multiflorum Thunb.: a review," Journal of Ethnopharmacology, vol. 159, pp. 158-183, 2015.

[6] J. Yu, J. Xie, X.-J. Mao et al., "Hepatoxicity of major constituents and extractions of radix polygoni multiflori and radix polygoni multiflori praeparata," Journal of Ethnopharmacology, vol. 137, no. 3, pp. 1291-1299, 2011.

[7] H. Dong, D. Slain, J. Cheng, W. Ma, and W. Liang, "Eighteen cases of liver injury following ingestion of polygonum multiflorum," Complementary Therapies in Medicine, vol. 22, no. 1, pp. 70-74, 2014.

[8] K. A. Jung, H. J. Min, S. S. Yoo et al., "Drug-induced liver injury: twenty five cases of acute hepatitis following ingestion of polygonum multiflorum thunb," Gut and Liver, vol. 5, no. 4, pp. 493-499, 2011.

[9] Q. Dong, N. Li, Q. Li et al., "Screening for biomarkers of liver injury induced by Polygonum multiflorum: a targeted 
metabolomic study," Frontiers in Pharmacology, vol. 6, article no. 217, 2015.

[10] X. Lei, J. Chen, J. T. Ren et al., "Liver damage associated with Polygonum multiflorum Thunb.: a systematic review of case reports and case series," Evidence-Based Complementary and Alternative Medicine, vol. 2015, Article ID 459749, 9 pages, 2015.

[11] W. Li, Y. Deng, R. Dai et al., "Chromatographic fingerprint analysis of Cephalotaxus sinensis from various sources by highperformance liquid chromatography-diodearray detectionelectrospray ionization-tandem mass spectrometry," Journal of Pharmaceutical and Biomedical Analysis, vol. 45, no. 1, pp. 3846, 2007.

[12] Y. Chen, Y. Yan, M. Y. Xie et al., "Development of a chromatographic fingerprint for the chloroform extracts of Ganoderma lucidum by HPLC and LC-MS," Journal of Pharmaceutical and Biomedical Analysis, vol. 47, no. 3, pp. 469-477, 2008.

[13] Z. Liu, Y. Liu, C. Wang et al., "Comparative analyses of chromatographic fingerprints of the roots of Polygonum multiflorum thunb. and their processed products using RRLC/DAD/ESIMSn," Planta Medica, vol. 77, no. 16, pp. 1855-1860, 2011. 


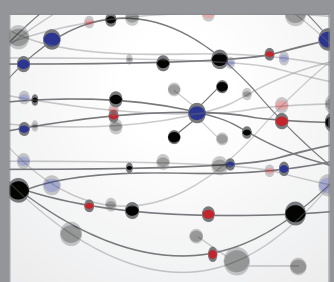

The Scientific World Journal
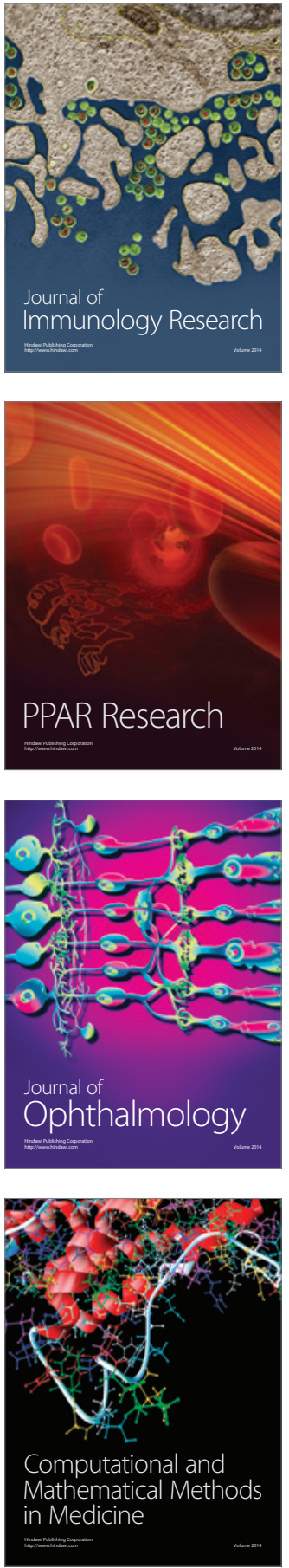

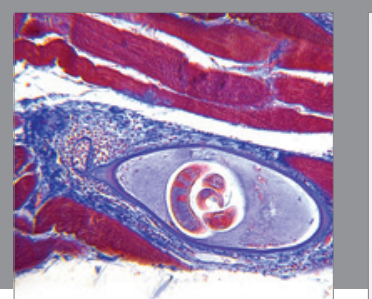

Gastroenterology Research and Practice
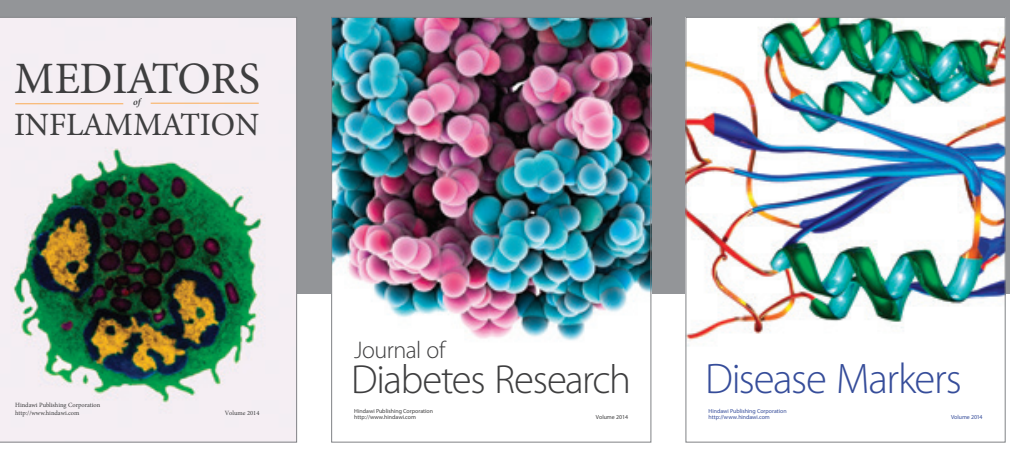

Disease Markers

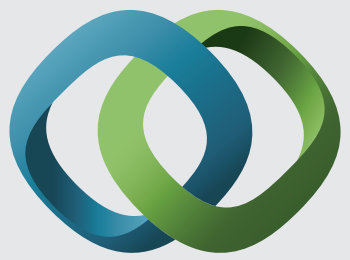

\section{Hindawi}

Submit your manuscripts at

https://www.hindawi.com
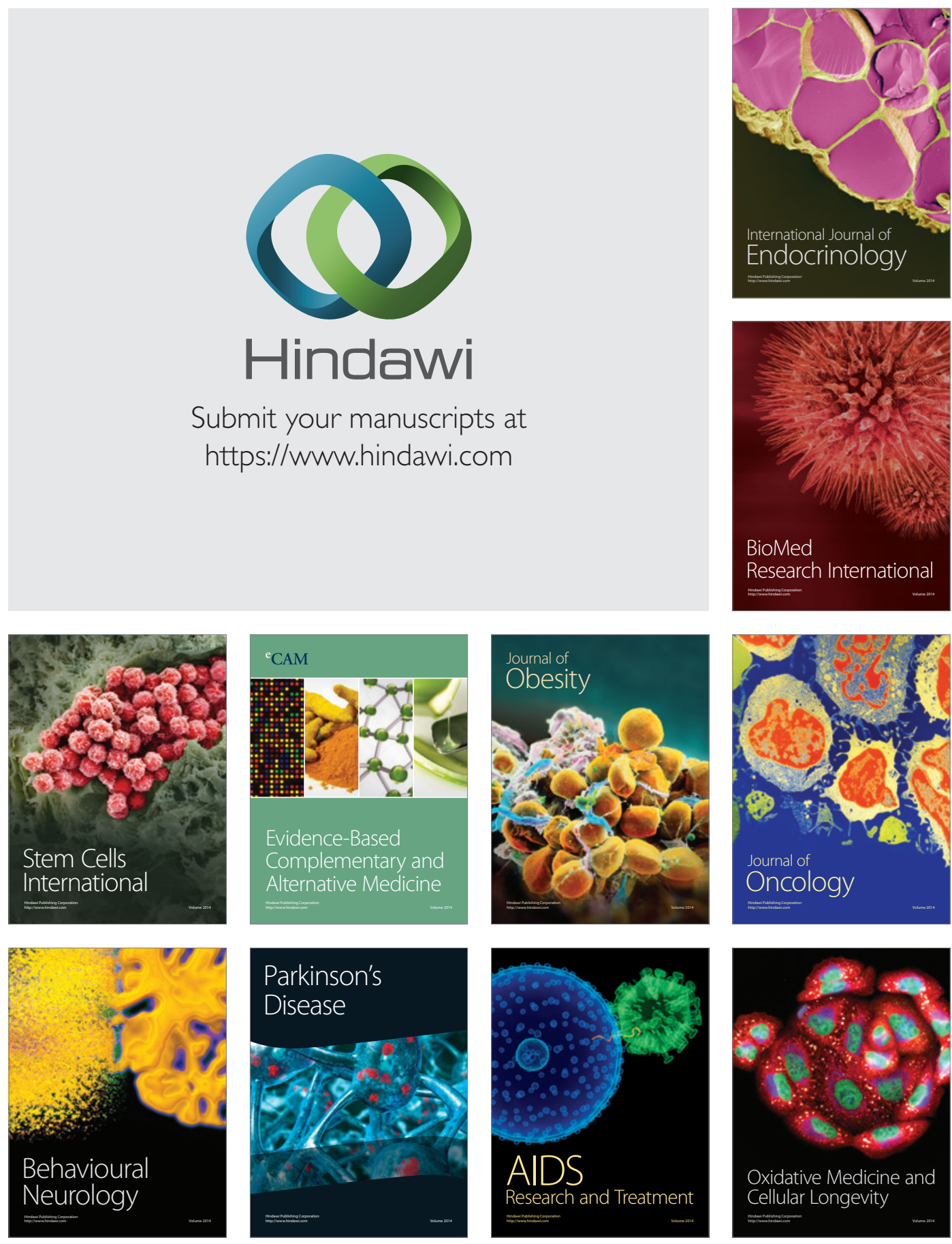